\section{From DNA to diversity: molecular genetics and the evolution of animal design (2nd ed.)}

\author{
SB Carroll, JK Grenier and SD Weatherbee \\ Blackwell Publishing Ltd, Oxford, UK; 2004. 258pp. £32.50, \\ paperback. ISBN 1405119500.
}

Heredity (2006) 96, 335. doi:10.1038/sj.hdy.6800800

\section{Reviewed by KL Hammond and TT Whitfield}

This book tackles a big question: how do genetic changes explain the diversity of animal form? To answer it, the authors draw together the disciplines of molecular genetics, genomics, phylogeny, palaeobiology and developmental biology to shed light on the molecular and developmental mechanisms that underlie evolutionary change. Despite this polymathic approach, the book is small enough to maintain a coherent story throughout (and to take on the train or read in the bath). It is aimed primarily at undergraduates or the nonspecialist, but will be enjoyed equally well by a more specialised audience; as developmental biologists with a strong interest in evolution, we found it both an enjoyable and informative read.

This book does not set out to be a comprehensive text; rather, it serves as a general introduction to the field, setting out key concepts and illustrating these with carefully chosen examples from across the animal kingdom. The salient principles are reinforced regularly, and the chosen examples give a real idea of the breadth and diversity of the field. The level of detail is sufficient for a full understanding of each topic, but never detracts from the general thread of the text, whereas the reference lists are comprehensive enough to allow you to follow-up any examples that have caught your fancy. Throughout, the text is supported by beautiful illustrations, ranging from wonderful photos of some of the most visually arresting examples in the field to excellent diagrams and flow charts.

The book begins by exploring the origins of the spectacular diversity in animal form. The account of fossil evidence here is tantalisingly brief, concentrating only on the origin and diversification of animal forms in the Cambrian. It would have been interesting to have a similar introduction to some of the other key transitions that are discussed later on in the book, such as the evolution of jaws in the vertebrate lineage. Instead, the emphasis quickly shifts to a developmental perspective, with a description of the basic genetic 'toolkit' from which all animals are constructed. The major focus here is the fruit fly; although developmental biologists could probably skip these chapters, they serve as a wonderful reminder of the beauty and logic of early Drosophila development.

Only when all the basics have been established does the book tackle the genetic changes that underlie morphological variation. The first examples of natural variation within a species are simple and familiar: coat colour variants of mammals, so popular among pet fanciers. These pigmentation differences are accounted for by polymorphic variants of the genes coding for proteins in the pigmentation pathway. We are then taken through examples of diversity between species, such as the loss of limbs from the basic segmented body plan of arthropods, giving rise to the limbless insect abdomen, and the molecular genetic changes in Hox expression that led to the diversification of insect wing appendages (from beetle elytra to the fly haltere). A section is devoted to novelties (the appearance of completely new structures, such as the turtle shell) and atavisms (a return to more ancestral forms).

The major thesis of the book, arrived at in the last few chapters, is that changes in gene regulatory sequencesrather than changes in protein coding regions-have driven changes in morphology. This is not a new idea, but is set nicely here in the context of the introduction provided by the previous chapters. Although recent comparative genomic data are taken into account, some considerations are perhaps lacking: an overview of genome structure and the range of genetic differences that distinguish species would have been helpful. Point mutations and small-scale duplications and rearrangements are discussed, and the evolution of the Hox complex is treated in some detail. However, larger scale chromosomal rearrangements, which have clearly occurred when we examine chromosome structure and gene order between different species, are not covered anywhere.

In the last few pages, the book returns to the debate that originally fuelled disagreement between Darwin and Bateson-does evolution occur in saltatory fashion, or is there a continual and gradual accumulation of smallscale changes? Although complimentary of Bateson's huge contributions to genetics, this book lands very much on the side of Darwin, concluding that small, gradual changes, such as we see within species, accumulated over millions of years, can lead to speciation. Sudden and dramatic changes of form, proposed by Bateson to be potential speciation events, are here considered as rare variants that would be eliminated by natural selection. The authors present a convincing argument, but are clear and honest in pointing out the caveats and assumptions in reaching their conclusion. Nevertheless, the question is still not answered with any certainty; even with genome data at our fingertips, we still do not understand all the secrets of evolution. The studies described in this book and those to come in the future will, bit by bit or perhaps in leaps and bounds, unravel this wonderful puzzle.

KL Hammond and TT Whitfield Centre for Developmental and Biomedical Genetics, Department of Biomedical Science, University of Sheffield, Sheffield S10 2TN, UK

E-mails: t.whitfield@sheffield.ac.uk or k.hammond@sheffield.ac.uk 